Abstract

\title{
Composition of Herpesvirus Ribonucleoprotein Complexes ${ }^{\dagger}$
}

\author{
Eric S. Pringle ${ }^{1,2, *}$ and Craig McCormick ${ }^{1,2}$ \\ 1 Department of Microbiology and Immunology, Dalhousie University, Halifax, NS B3H 4R2, Canada; \\ craig.mccormick@dal.ca \\ 2 Beatrice Hunter Cancer Research Institute, Halifax, NS B3H 4R2, Canada \\ * Correspondence: eric.pringle@dal.ca \\ + Presented at Viruses 2020-Novel Concepts in Virology, Barcelona, Spain, 5-7 February 2020.
}

Published: 4 July 2020

\begin{abstract}
Herpesvirus genomes are decoded by host RNA polymerase enzymes, generating messenger ribonucleotides (mRNA) that are post-transcriptionally modified and exported to the cytoplasm through the combined work of host and viral factors. These viral mRNA bear $5^{\prime}-\mathrm{m}^{7} \mathrm{GTP}$ caps and poly(A) tails that should permit the assembly of canonical host eIF4F cap-binding complexes to initiate protein synthesis. However, the precise mechanisms of translation initiation remain to be investigated for Kaposi's sarcoma-associated herpesvirus (KSHV) and other herpesviruses. During KSHV lytic replication in lymphoid cells, the activation of caspases leads to the cleavage of eIF4G and depletion of eIF4F. Translating mRNPs depleted of eIF4F retain viral mRNA, suggesting that non-eIF4F translation initiation is sufficient to support viral protein synthesis. To identify proteins required to support viral protein synthesis, we isolated and characterized actively translating messenger ribonucleoprotein (mRNP) complexes by ultracentrifugation and sucrose-gradient fractionation followed by quantitative mass spectrometry. The abundance of host translation initiation factors available to initiate viral protein synthesis were comparable between cells undergoing KSHV lytic or latent replication. The translation initiation factors eIF4E2, NCBP1, eIF4G2, and eIF3d were detected in association with actively translating mRNP complexes during KSHV lytic replication, but their depletion by RNA silencing did not affect virion production. By contrast, the N6-methyladenosine methyltransferase METTL3 was required for optimal late gene expression and virion production, but was dispensable for genome replication. Furthermore, we detected several KSHV proteins in actively translating mRNP complexes that had not previously been shown to play roles in viral protein synthesis. We conclude that KSHV usurps distinct host translation initiation systems during latent and lytic phases of infection.
\end{abstract}

(C) 2020 by the authors. Licensee MDPI, Basel, Switzerland. This article is an open access article distributed under the terms and conditions of the Creative Commons Attribution (CC BY) license (http://creativecommons.org/licenses/by/4.0/). 